\title{
Structural characterization and evolutionary analyses of the Coccidioides immitis and Coccidioides posadasii mitochondrial genomes
}

1 Marcus de Melo Teixeira ${ }^{1,2^{*}}$, B. Franz Lang ${ }^{3 *}$, Daniel R. Matute ${ }^{4}$, Jason E. Stajich ${ }^{5,6}$, Bridget

2 Barker, ${ }^{1}$,

$4 \quad{ }^{1}$ Pathogen and Microbiome Institute, Northern Arizona University, Flagstaff, AZ, USA

$5 \quad{ }^{2}$ Faculty of Medicine, University of Brasília-DF, Brazil

$6{ }^{3}$ Robert Cedergren Centre for Bioinformatics and Génomiques, Département de Biochimie,

7 Université de Montréal, Montréal-QC, Canada

$8{ }^{4}$ Department of Biology, University of North Carolina, Chapel Hill, USA.

$9{ }^{4}$ Institute for Integrative Genome Biology, University of California, Riverside, CA, 92521;

$10{ }^{5}$ Department of Microbiology and Plant Pathology, University of California, Riverside, CA, 92521;

$12 *$ These authors contributed equally to the manuscript

$13 @$ Correspondence:

14 Bridget M. Barker, bridget.barker@,nau.edu. Pathogen and Microbiome Institute, Northern Arizona

15 University, Applied Research \& Development Building, 1395 S. Knoles Drive, Flagstaff, Arizona

$16 \quad 86011-4073$

17 Keywords: Coccidioides, coccidioidomycosis, mitochondrial, introns group I and II.

18 ORCIDs:

19 MMT: 0000-0003-17633464

20 BFL: 0000-0003-1035-5449

21 DRM: 0000-0002-7597-602X

22 JES: 0000-0002-7591-0020

23 BMB: 0000-0002-3439-4517 


\section{Abstract}

25 Fungal mitochondrial genomes encode for genes involved in crucial cellular processes, such as

26 oxidative phosphorylation and mitochondrial translation, and these genes have been used as

27 molecular markers for population genetics studies. Coccidioides immitis and C. posadasii are

28 endemic fungal pathogens that cause coccidioidomycosis in arid regions across both American

29 continents. To date, almost one hundred Coccidioides strains have been sequenced. The focus of

30 these studies has been exclusively to infer patterns of variation of nuclear genomes (nucDNA).

31 However, their mitochondrial genomes (mtDNA) have not been studied. In this report, we describe

32 the assembly and annotation of mitochondrial reference genomes for two representative strains of $C$.

33 posadasii and $C$. immitis, as well as assess population variation among 77 published genomes. The

34 circular-mapping mtDNA molecules are $68.2 \mathrm{~Kb}$ in C. immitis and $75.1 \mathrm{~Kb}$ in C. posadasii. We

35 identified the fourteen mitochondrial protein-coding genes common to most fungal mitochondria,

36 including genes encoding the small and large ribosomal RNAs ( $r n s$ and $r n l)$, the RNA subunit of

37 RNAse $\mathrm{P}(r n p \mathrm{~B})$, and 26 tRNAs organized in polycistronic transcription units, which are mostly

38 syntenic across different populations and species of Coccidioides. Both Coccidioides species are

39 characterized by a large number of group I and II introns, harboring twice the number of elements as

40 compared to closely related Onygenales. The introns contain complete or truncated ORFs with high

41 similarity to homing endonucleases of the LAGLIDADG and GIY-YIG families. Phylogenetic

42 comparison of the mtDNA and nucDNA genomes shows discordance, possibly due to differences in

43 patterns of inheritance. In summary, this work represents the first complete assessment of

44 mitochondrial genomes among several isolates of both species of Coccidioides, and provides a

45 foundation for future functional work. 


\section{Introduction}

48 Fungal mitochondrial genomes exist as either linear or circular-mapping molecules and range from

$49 \sim 17.6 \mathrm{~kb}$ (e.g. Schizosaccharomyces pombe Genbank ID MK618090.1) to well over $200 \mathrm{~kb}$ (e.g.

$50 \quad 272,238$ bp in Morchella importuna (1)). Fungal mitochondrial genomes usually encode proteins

51 involved in oxidative phosphorylation - the main source of ATP production of the cell - as well as

52 two ribosomal RNA subunits, and a set of tRNAs involved in mitochondrial ribosome translation.

53 More specifically, fungal mitochondrial protein-coding genes fall into several classes: seven subunits

54 of ubiquinone oxidoreductase ( $\mathrm{nad}$; not present in a number of Saccharomycotina and in fission

55 yeasts, (2)), cytochrome b (cob), three subunits of cytochrome oxidase (cox) and up to three ATP

56 synthase subunits (atp; the presence of atp 8 and atp9 varies among fungal taxa) (3). Also, a gene

57 encoding a ribosomal protein subunit (rps3) is present in most fungal mitochondrial genomes.

58 Mitochondrial protein-coding genes are frequently intercalated with genes that encode structural

59 RNAs: ribosomal RNAs (small and large subunit rRNAs $r n s$ and $r n l$ ), the RNA subunit of RNase P

$60(\mathrm{rnpB})$ with infrequent occurrence across fungi, and variable numbers of tRNAs. Notable exceptions

61 are the nad genes, which tend to be organized in operon-like structures, with some of the genes

62 overlapping without discernable intergenic regions (e.g., nad4L situated upstream of nad5,

63 overlapping by one to a dozen or more nucleotides) (3).

65 are inserted in protein-coding as well as rRNA genes (4). For instance, Endoconidiophora species 
67 especially when transcriptome data are not available. Both intron groups may contain complete or

68 truncated ORFs that encode either homing endonucleases of the LAGLIDADG and GIY-YIG

69 families, or reverse transcriptases/maturases (6). If present, these proteins direct an intron transfer

70 within mitochondrial genomes of genetically compatible fungal isolates, or less frequently across

71 genera, and even kingdom boundaries (7). Mitochondrial DNA (mtDNA)-encoded genes are

72 particularly prone to crossing species boundaries. As intron transfer via homing endonucleases

73 involves genetic co-conversion of flanking exon sequences, phylogenetic inferences using mtDNA-

74 especially genes with high intron numbers (e.g., coxl, cob and $r n l(3,8)$ ) - may reveal replacement

75 of coding regions, related to ongoing intron invasion.

In this study, we focus on describing the mitogenomes of Coccidioides immitis and $C$.

posadasii (Ascomycota, Onygenales), which are fungal species endemic to both American

continents, and the causative agents of coccidioidomycosis (9). This disease is most frequently

79 reported in the "Lower Sonoran Life Zone" in California, Arizona, Texas, and northwestern Mexico

80 (10). However, the disease is also reported in arid and semi-arid areas throughout the American

81 continents (11). The two species have a complex evolutionary history dominated by biogeographic

82 distribution patterns $(12,13)$. Coccidioides immitis has been found in California and Baja Mexico as

83 well in eastern Washington state, and each region harbors unique genotypes (14-16). Coccidioides

84 posadasii is present throughout Arizona, Texas, Central, and South America, and population

85 structure has been described as containing an Arizona population, a Texas/Mexico/South America

86 (TX/MX/SA) population, and a distinct Caribbean population (13). 
Notably, nucDNA studies have found extensive differentiation between species of

88 Coccidioides with some evidence for gene flow between species $(17,18)$. The two species, $C$.

89 immitis and C. posadasii, can be discriminated based on polymorphisms found at the first intron of

90 the coxl gene (19). Yet, no studies have addressed whether or not mtDNA reflects the divergence of

91 ncDNA, or if mtDNA has moved between Coccidioides species or among populations. In this study

92 we: $i$ ) describe the full circular-mapping mitogenomes of $C$. posadasii and C. immitis, $i$ ) compare

93 their core genes, structural RNAs and introns of group I and II with other Onygenales fungal species,

94 and iii) compare the evolutionary trajectories between the mtDNA and nucDNA genomes among

95 publicly available genomes of this medically important fungal pathogen.

96 Materials and Methods

\section{Mitochondrial genome assembly and annotation}

98 Paired end Illumina sequence reads from 20 Coccidioides immitis and 57 C. posadasii were retrieved

99 from the Sequence Read Archive (SRA) and accessions and details are listed in Table S1. Following

100 cleaning and quality-clipping of reads with Trimmomatic v0.35, we assembled the genomes of $C$.

101 posadasii Tucson-2 and C. immitis WA221 using the SPAdes Genome Assembler v3.14.0 (20) with

102 a kmer sizes 61,91 , and 127 . We identified mitochondrial contigs in this initial assembly using

103 similarity searches with expected fungal genes. To minimize assembly error we (i) used Rcorrector

104 [Song, L., Florea, L. Rcorrector: efficient and accurate error correction for Illumina RNA-seq reads.

105 GigaSci 4, 48 (2015).] for read correction, (ii) reduced the number of Illumina reads to a target kmer

106 coverage of the mtDNA between 30-50x, (iii) reads mapping against the identified mitochondrial

107 contigs were identified with Bowtie2 (21), which were then (iii) reassembled with Spades, resulting

108 in preliminary (uncorrected) mitogenome assemblies. In a final step, all reads of the reduced 30-50x 
109 read set were aligned back to the preliminary assembly with Bowtie 2 and analyzed for kmer coverage

110 with Bedtools v2.29.2 (22). We identified incorrectly-assembled reads, defined by kmer frequency

111 values of two or lower (likely the result of hybrid reads, originating from ligation of unrelated

112 genomic DNA fragments during library construction), and removed them from the final assemblies.

113 For both species, we obtained single circular-mapping closed contigs that carry the expected full set

114 of fungal mitochondrial genes.

115 To compare the Coccidioides mtDNA assembles with other fungi, we retrieved full mitochondrial

116 assemblies from other Onygenales available in the NCBI GenBank database: Histoplasma

117 capsulatum H143 (GG692467.1, direct submission) Paracoccidioides brasiliensis Pb18

118 (AY955840.1, (23)), Blastomyces dermatitidis ATCC 18188 (GG753566.1, direct submission),

119 Epidermophyton flocossum ATCC 26072 (AY916130.1, (24)), Trichophyton rubrum BMU 01672

120 (FJ385026.1, (25)) and Ascosphaera apis ARSEF 7405 (AZGZ01000045.1, (26)). Other close

121 relatives of Coccidioides (e.g. Uncinocarpus) had incomplete mitogenomes (27).

122 Mitochondrial genes as well as introns of group I and group II, tRNAs, RNase P RNA ( $r n p B$ ), and

123 the small and large subunit rRNAs ( $r n s$ and $r n l$ ) for Coccidioides and other related Onygenalean

124 fungi were annotated using the MFannot pipeline (https://megasun.bch.umontreal.ca/cgi-

125 bin/dev_mfa/mfannotInterface.pl; https://github.com/BFL-lab/Mfannot). Coccidioides annotations

126 were manually inspected and intron boundaries were checked and adjusted by aligning available

127 RNAseq data (27) with respective mitochondrial assemblies using Bowtie 2 (21). The assemblies and

128 annotations were deposited in GenBank (accession numbers TBD) and were visualized with the

129 OGDRAW pipeline (28).

130 Single nucleotide polymorphism assessment and phylogenetic analysis 
131 SNPs from 77 Coccidioides isolates were identified among the mitochondrial genomes. We mapped

132 Illumina paired-end reads into individual mitochondrial coding-genes using Burrows-Wheeler

133 Aligner (BWA) v 0.7.7 (29) to assembled mitochondrial references $C$. posadasii strain Tucson2 or $C$.

134 immitis strain WA221. Indels were realigned to its reference genomes using GATK

135 RealignerTargetCreator and IndelRealigner tools (GATK toolkit v 3.3-0 (30)). To call SNPs, we used

136 the UnifiedGenotyper package. We only included SNPs not located in potentially duplicated loci (as

137 identified by NUCmer, (31)), with more than 10X coverage, and with a minor allele frequency of at

138 least $10 \%$. We used the same approach to call SNPs for the nucDNA genomes (13). We generated

139 Maximum Likelihood (ML) concatenated trees for mtDNA and nucDNA using methods

140 implemented in IQ-TREE software (32) using -m MFP option (ModelFinder - (33)) for model

141 selection and 1,000 ultrafast bootstraps coupled with Shimodaira-Hasegawa-like approximate

142 likelihood ratio test (SH-aLRT) were performed for branch confidence test (34).Finally, we

143 compared the topology of the two trees using FigTree v1.4.2 -

144 http://tree.bio.ed.ac.uk/software/figtree/, and scored the disagreements the two topologies were using

145 TOPD/FMTS v $4.6(35)$.

\section{Results}

\section{The Coccidioides spp. mitogenome}

148 We assembled complete circular mtDNA molecules for each of the two species of Coccidioides. The

149 two assemblies differ in size: the mtDNA genome is $68.6 \mathrm{~Kb}$ in $C$. immitis and $75.1 \mathrm{~Kb}$ in $C$.

150 posadasii (Figure 1). There is variation in mtDNA genome size among Onygenales (Table 1). The

151 mtDNA of both species of Coccidioides are on the larger end of the continuum. The mitogenomes of

152 Coccidioides harbor 14 protein-coding genes responsible for the formation of ubiquinone

153 oxidoreductase, cytochrome b, cytochrome oxidase and ATP synthase protein complexes (Figure 1,

154 Figure 2). The two ribosomal small and large subunit rRNA genes ( $r n s$ and $r n l)$, RNase P RNA 
$155(\operatorname{rnp} B)$ and 26 tRNAs organized in polycistronic transcription units are all present. The gene

156 composition and synteny are conserved between Coccidioides (Onygenaceae), Blastomyces,

157 Histoplasma, and Paracoccidioides (all Ajellomycetaceae) (Figure 1, (36)). The position of the gene

158 atp 8 differs between the Onygenaceae/Ajellomycetaceae species and other species of the

159 Onygenales, such as dermatophytes (Trichophyton rubrum and Epidermophyton floccosum -

160 Arthrodermataceae), and the bee-pathogenic fungus Ascosphaera apis (Ascosphaeraceae, Figure 2).

161 We observed no gene gain and losses of core mitochondrial genes within Onygenalean fungi (Figure 162 2).

The large size of the mtDNA genome in Coccidioides is due to the presence of introns and

164 intron-encoded open reading frames (ORFs) in both Coccidioides species, resulting in a dramatic

165 increase of intron type I and intron type II (Table 1, Figure 1) compared to other Ajellomycetaceae

166 fungi. In fact, Coccidioides harbors twice the number of elements found in B. dermatitidis. The

167 dermatophyte genera, Epidermophyton and Trichophyton, have only six and two intron elements

168 respectively, whereas $C$. immitis and C. posadasii contain 39 elements respectively (Table 1). The

169 introns found in the Coccidioides mitogenomes contain complete or truncated ORFs with high

170 similarity to homing endonucleases of the LAGLIDADG and GIY-YIG families (Table 1). Both

171 species contain 15 complete copies of Intron IB (Table 1). Ascopharaceae apis also has a large

172 mtDNA genome $(118.65 \mathrm{~Kb})$ and a high number of intron-type I, specifically the intron I - derived,

173 B1 element (Table 1). The frequency and distribution of intron-types I and II in the genes nadh5, cob

174 and coxl differ between C. immitis and C. posadasii, but these features are not differentiated within

175 the species-complexes (Figure 1, Figure 2).

176 Comparing mtDNA and nucDNA whole-genome trees 
177 Finally, we compared the mitochondrial phylogeny with the whole genome species phylogeny. To

178 score the differences in partitions produced between mtDNA and nucDNA trees, we used TOPD

179 (35). If two trees are completely congruent the split distance score is 0 , whereas with complete tree

180 disagreement the score is 1 . Differences are due to dissimilarity between the topologies as well as the

181 number of overlapping taxa. The split distance is the ratio of (different/possible) between the

182 Coccidioides mtDNA and nucDNA phylogenetic tree is $0.89(132 / 148)$, thus indicating that overall

183 topologies are consistent. Both topologies support species divergence between $C$. immitis and $C$.

184 posadasii (Figure 3). The mtDNA tree topology, shows a clear distinction between $C$. immitis and $C$.

185 posadasii with no isolates being assigned to a different species. This results suggest no mtDNA gene

186 exchange.

187 Those populations have low intraspecific genetic variation, suggesting either strict clonal dispersion, 188 or a recent founder effect (13).

Even though the main shape of the two topologies were consistent with each other. We also

observed differences. Consistent with previous results, the nucDNA tree topology revealed three $C$.

191 posadasii monophyletic populations: Arizona, Mexico/Texas/South America (MX/TX/SA) and

192 Caribbean (13). Based on mtDNA among the three main C. posadasii populations that have been

193 previously defined by markers differ substantially (Figure 3). Specifically, the mtDNA phylogeny

194 shows C. posadasii Arizona, Texas/Mexico/South America, and Caribbean clades are paraphyletic,

195 and individuals from these previously defined populations are dispersed into multiple clades in the

196 mitogenome tree (Figure 3). First, the clade Mito 1 harbors 16 isolates in which 13 concordantly

197 belong to the Arizona population using nucDNA genomes. The isolates B10813, Tucson2 and

198 Tucson20 (AZ clade I) have conflicting phylogenetic distributions, previously these placed within

199 Texas/Mexico/SouthAmerica and AZ clade I (Figure 3). Second, the clade Mito II contains the

200 Venezuela group and three strains from the Arizona population (Tucson3, Tucson4 and Tucson14), 
201 although the Venezuela mitochondrial and nucDNA topologies are congruent. The next clade of $C$.

202 posadasii is composed of four strains from Guatemala, and is perfectly concordant with the nucDNA

203 tree. The clade Mito III is composed of two isolates from the AZ Clade I (Tucson6 and Tucson16)

204 and two others from the Caribbean clade, and this lineage also shows conflicting phylogenetic

205 placement (Figure 3). Finally, the clade Mito IV is composed primarily of isolates from the

206 Texas/Mexico/South America clade.

Both nucDNA and mtDNA phylogenies revealed a clade composed of strains from Washington (16) which is genetically distinct from the rest of $C$. immitis (Figure 3). No other consistent pattern of clustering was observed for the remaining $C$. immitis individuals comparing the

210 two phylogenies (Figure 3).

211 In general, our results suggest that nucDNA and mtDNA have similar evolutionary

212 trajectories with no evidence of interspecific mtDNA exchange, but also the existence of

213 phylogenetic incongruence at recent scales possibly due to within-species recombination.

\section{Discussion}

215 Analysis of mitochondrial genomes as molecular markers confirms that the Coccidioides genus

216 contains two species: C. immitis and C. posadasii. Mitochondrial markers are extensively used as

217 molecular markers in speciation studies, including for Coccidioides $(19,37,38)$. However molecular

218 systematics of Coccidioides based on mitochondrial genes may lead to ambiguous conclusions at an

219 intraspecies population level. Interestingly, both the mtDNA phylogeny and admixture plots of two

220 distinct and divergent populations C. immitis Washington and C. posadasii Venezuela clearly reveal

221 monophyly, as they are both reciprocally homozygous and no mixed genotypes are found (Figure 3).

222 Both of these populations have emerged within the last 6,000 years according to estimates $(13,16)$. 
223 This suggests a strong founder effect followed by asexual reproduction in two endemic areas of the

224 disease (39).

The evolutionary trajectories of both mtDNA and nucDNA genomes have been investigated

using next generation sequencing data. Certainly, mtDNA and nucDNA genotypic incompatibilities

may exist, as well as undetermined effects of cross-species hybridization and introgression (40). This

is due in part to the fact that mitochondrial replication and division is not synchronized with nuclear

division, and cells can contain numerous mitochondria, which may not undergo genetic

recombination and may increase in number without cell division (41). Conflicting phylogenetic and

population distributions have been observed in other pathogenic fungi, and our results indicate shared

ancestry among recently diverged $C$. immitis and $C$. posadasii populations. For example,

Paracoccidioides brasiliensis and $P$. restrepiensis appear to be polyphyletic using mtDNA markers,

and the tree topologies differ from those obtained from nucDNA markers (42). Moreover, it is

suggested that mitochondrial interspecific hybridization and introgression occurs in Paracoccidioides

(42). Mitochondrial genomes can be difficult to assemble if high heterozygosity exists, as observed

for the opportunistic pathogen Candida metapsilosis, which is part of the C. parapsilosis complex

(43). This novel pathogen is a result of a hybridization event, which was detected in part by

analyzing the mitochondrial genome. Within the primary pathogen Cryptococcus gattii complex,

240 incongruences between mitochondrial and nuclear genes have been also reported (8). Mitochondrial

241 genotypic and consequent phenotypic variation among these pathogen complexes is associated with

242 virulence traits (44). These patterns are not restricted to human fungal pathogens. Some strains of the

243 plant fungal pathogen Verticillium longisporum present a mosaic mitochondrial genome structure due

244 to bi-parental inheritance impacting niche adaptation (45). Population genomic analyses of the

245 lichen-forming fungi Rhizoplaca melanophthalma species complex suggest that hybridization and 
recombination in mitochondria might play a role in the speciation process of these symbiotic fungi

247 (46).

249 lifestyles (47) leading us to hypothesize that virulence, thermo-adaptation and oxidative stress could

250 be driving genetic differentiation in mtDNA in Coccidioides species and populations. For example,

251 in Saccharomyces, specific mutations the coxl gene in the mtDNA are associated with adaptation to

252 variable temperatures. The authors suggest that the yeast mitochondrial genome is a hotspot in the

253 evolution of thermal adaptation in Saccharomyces species $(48,49)$. C. posadasii is more heat tolerant

254 than C. immitis and private alleles found in the mitochondrial genome might be responsible for this

255 interspecific phenotypic variation. Importantly, in this manuscript we provided high-quality

256 assemblies and annotations for the Coccidioides mitogenomes, which will facilitate deeper

257 investigations into the impact of mitochondrial evolution in Coccidioides ' niche adaptation, with

258 particular emphasis on mammalian host co-evolution and oxidative stress responses.

\section{Tables}

261 Table 1. Numbers of introns and classes among Onygenalean fungi

\begin{tabular}{|c|c|c|c|c|c|c|c|c|}
\hline & $\begin{array}{l}\text { C. } \\
\text { immitis }\end{array}$ & $\begin{array}{l}\text { C. } \\
\text { posadasii }\end{array}$ & $\begin{array}{l}\text { H. } \\
\text { capsulatum }\end{array}$ & $\begin{array}{l}P . \\
\text { brasiliensis }\end{array}$ & $\begin{array}{l}\text { B. } \\
\text { dermatitidis }\end{array}$ & $\begin{array}{l}\text { E. } \\
\text { flocossum }\end{array}$ & $\begin{array}{l}T . \\
\text { rubrum }\end{array}$ & A. apis \\
\hline $\begin{array}{l}\text { Mitochondrial } \\
\text { genome size (bp) }\end{array}$ & 68,597 & 75,194 & 39,129 & 71,335 & 51,071 & 30,910 & 26,985 & 118,650 \\
\hline $\begin{array}{l}\text { Intron IB } \\
\text { (complete) }\end{array}$ & 15 & 15 & 3 & 5 & 6 & 3 & 0 & 8 \\
\hline $\begin{array}{l}\text { intron IB (extra } \\
\text { insertion) }\end{array}$ & 0 & 0 & 1 & 0 & 0 & 0 & 0 & 0 \\
\hline $\begin{array}{l}\text { intron IB (5', } \\
\text { partial) }\end{array}$ & 0 & 1 & 0 & 0 & 0 & 0 & 0 & 1 \\
\hline $\begin{array}{l}\text { intron IB (3', } \\
\text { partial) }\end{array}$ & 1 & 2 & 1 & 0 & 2 & 0 & 0 & 1 \\
\hline intron IA & 3 & 3 & 1 & 0 & 1 & 1 & 2 & 1 \\
\hline $\begin{array}{l}\text { intron IA ( } 5^{\prime}, \\
\text { partial) }\end{array}$ & 0 & 1 & 0 & 0 & 0 & 0 & 0 & 1 \\
\hline $\begin{array}{l}\text { intron I (derived, } \\
\text { A) }\end{array}$ & 1 & 1 & 0 & 0 & 2 & 1 & 0 & 5 \\
\hline $\begin{array}{l}\text { intron I (derived, } \\
\text { B1) }\end{array}$ & 2 & 2 & 0 & 4 & 0 & 0 & 0 & 11 \\
\hline intron ID & 4 & 3 & 1 & 0 & 1 & 1 & 0 & 5 \\
\hline
\end{tabular}




\begin{tabular}{|c|c|c|c|c|c|c|c|}
\hline intron IC1 & 0 & 0 & 0 & 0 & 0 & 0 & 0 \\
\hline intron IC2 & 4 & 4 & 0 & 1 & 1 & 0 & 0 \\
\hline $\begin{array}{l}\text { intron I (derived, } \\
\text { B2) }\end{array}$ & 1 & 1 & 1 & 0 & 1 & 0 & 0 \\
\hline $\begin{array}{l}\text { intron II } \\
\text { (domainV) }\end{array}$ & 4 & 5 & 1 & 3 & 2 & 0 & 0 \\
\hline intron II, derived & 1 & 1 & 0 & 0 & 0 & 0 & 0 \\
\hline Total & 36 & 39 & 9 & 13 & 16 & 6 & 2 \\
\hline
\end{tabular}

\section{Figure Legends}

264 Figure 1 - Circular maps of C. immitis and C. posadasii mitogenomes. The assembled and annotated

265 genome features were converted into Genebank format and loaded into the OGDraw pipeline for

266 physical visualization of the coding and non-coding elements of the mitochondrial genomes.

267 Figure 2 - Mitochondrial gene content and synteny among Onygenalean fungi. Genes as color-coded

268 (see legend) and displayed according positioning on the genome. The mitogenomes genomes are

269 highly syntenic but atp 8 gene positioning is divergent between those fungal families.

270 Figure 3 - Tree topology comparisons of Coccidioides nucDNA (left panel) and mtDNA (right

271 panel) phylogenomic trees. Phylogenetic tree branches are proportional to the nucleotide divergence

272 (see scale) and the main clades are highlighted. Bootstrap support was calculated, and branch support

273 was added to the corresponding clade. The terminal taxa are color-coded according to their placement

274 on the nucDNA tree and taxa are connected between mtDNA and nucDNA phylogenomic trees in

275 order to visualize concordance (solid lines) vs discordance (dotted lines).

\section{Conflict of Interest}

277 The authors declare that the research was conducted in the absence of any commercial or financial

278 relationships that could be construed as a potential conflict of interest.

\section{Author Contributions}

280 The manuscript was written and edited by MT, JES, BFL, DRM and BMB. Data was analyzed by

281 MT and BFL. Funding provided by BMB.

\section{Funding}


283 BFL was supported by the Natural Sciences and Engineering Research Council of Canada (NSERC;

284 RGPIN- 2017-05411) and by the 'Fonds de Recherche Nature et Technologie', Quebec.

285 JES is a CIFAR Fellow in the program Fungal Kingdom: Threats and Opportunities and was 286 supported by University of California MRPI grants MRP-17-454959 "UC Valley Fever Research

287 Initiative" and VFR-19-633952 "Investigating fundamental gaps in Valley Fever knowledge" and

288 United States Department of Agriculture - National Institute of Food and Agriculture Hatch Project

289 CA-R-PPA-5062-H.

290 BMB was supported by NIH/NIAID R21 AI128536, State of Arizona TRIF funding, and Flinn

291 Foundation.

292 DRM was supported by NIH R01GM121750.

293

\section{Acknowledgments}

295 We thank The Translational Genomics Institute and the Pathogen and Microbiome Institute and

296 Northern Arizona University computing resources for data processing support. Special thanks to Dr.

297 John Taylor for helpful discussion and edits.

\section{Supplementary Material}

299 Accessions and details are listed in Table S1. 


\section{References}

301 1. Liu W, Cai Y, Zhang Q, Chen L, Shu F, Ma X, Bian Y. 2020. The mitochondrial genome of Morchella importuna $(272.2 \mathrm{~kb})$ is the largest among fungi and contains numerous introns,

3. Aguileta G, de Vienne DM, Ross ON, Hood ME, Giraud T, Petit E, Gabaldon T. 2014. High

4. Pogoda CS, Keepers KG, Nadiadi AY, Bailey DW, Lendemer JC, Tripp EA, Kane NC. 2019.

5. Zubaer A, Wai A, Hausner G. 2018. The mitochondrial genome of Endoconidiophora Genome streamlining via complete loss of introns has occurred multiple times in lichenized fungal mitochondria. Ecol Evol 9:4245-4263.

6. Lang BF, Laforest MJ, Burger G. 2007. Mitochondrial introns: a critical view. Trends Genet 23:119-25.

7. Stoddard BL. 2011. Homing endonucleases: from microbial genetic invaders to reagents for targeted DNA modification. Structure 19:7-15.

8. Bovers M, Hagen F, Kuramae EE, Boekhout T. 2009. Promiscuous mitochondria in Cryptococcus gattii. FEMS Yeast Research 9:489-503.

9. Barker BM, Litvintseva AP, Riquelme M, Vargas-Gastelum L. 2019. Coccidioides ecology and genomics. Med Mycol 57:S21-S29.

10. Lacy GH, Swatek FE. 1974. Soil ecology of Coccidioides immitis at Amerindian middens in California. Appl Microbiol 27:379-88.

11. Kollath DR, Miller KJ, Barker BM. 2019. The mysterious desert dwellers: Coccidioides immitis and Coccidioides posadasii, causative fungal agents of coccidioidomycosis. Virulence 10:222-233.

12. Fisher MC, Koenig GL, White TJ, San-Blas G, Negroni R, Alvarez IG, Wanke B, Taylor JW. 2001. Biogeographic range expansion into South America by Coccidioides immitis mirrors New World patterns of human migration. Proc Natl Acad Sci U S A 98:4558-62.

13. Teixeira MM, Alvarado P, Roe CC, Thompson GR, 3rd, Patane JSL, Sahl JW, Keim P, Galgiani JN, Litvintseva AP, Matute DR, Barker BM. 2019. Population Structure and Genetic Diversity among Isolates of Coccidioides posadasii in Venezuela and Surrounding Regions. mBio 10 .

14. Teixeira MM, Barker BM, Stajich JE. 2019. Improved Reference Genome Sequence of Coccidioides immitis Strain WA_211, Isolated in Washington State. Microbiol Resour Announc 8.

15. McCotter OZ, Benedict K, Engelthaler DM, Komatsu K, Lucas KD, Mohle-Boetani JC, Oltean H, Vugia D, Chiller TM, Sondermeyer Cooksey GL, Nguyen A, Roe CC, Wheeler C, Sunenshine R. 2019. Update on the Epidemiology of coccidioidomycosis in the United States. Med Mycol 57:S30-S40. 
16. Engelthaler DM, Roe CC, Hepp CM, Teixeira M, Driebe EM, Schupp JM, Gade L, Waddell

19. Hamm PS, Hutchison MI, Leonard P, Melman S, Natvig DO. 2019. First Analysis of Human Coccidioides Isolates from New Mexico and the Southwest Four Corners Region: Implications for the Distributions of C. posadasii and C. immitis and Human Groups at Risk. J Fungi (Basel) 5.

20. Bankevich A, Nurk S, Antipov D, Gurevich AA, Dvorkin M, Kulikov AS, Lesin VM, Nikolenko SI, Pham S, Prjibelski AD, Pyshkin AV, Sirotkin AV, Vyahhi N, Tesler G, Alekseyev MA, Pevzner PA. 2012. SPAdes: a new genome assembly algorithm and its applications to single-cell sequencing. J Comput Biol 19:455-77.

21. Langmead B, Salzberg SL. 2012. Fast gapped-read alignment with Bowtie 2. Nat Methods 9:357-9.

22. Quinlan AR. 2014. BEDTools: The Swiss-Army Tool for Genome Feature Analysis. Current Protocols in Bioinformatics 47:11.12.1-11.12.34.

23. Cardoso MA, Tambor JH, Nobrega FG. 2007. The mitochondrial genome from the thermal dimorphic fungus Paracoccidioides brasiliensis. Yeast 24:607-16.

24. Tambor JH, Guedes RF, Nobrega MP, Nobrega FG. 2006. The complete DNA sequence of the mitochondrial genome of the dermatophyte fungus Epidermophyton floccosum. Curr Genet 49:302-8.

25. Wu Y, Yang J, Yang F, Liu T, Leng W, Chu Y, Jin Q. 2009. Recent dermatophyte divergence revealed by comparative and phylogenetic analysis of mitochondrial genomes. BMC Genomics 10:238.

26. Shang Y, Xiao G, Zheng P, Cen K, Zhan S, Wang C. 2016. Divergent and Convergent Evolution of Fungal Pathogenicity. Genome Biol Evol 8:1374-87.

27. Whiston E, Zhang Wise H, Sharpton TJ, Jui G, Cole GT, Taylor JW. 2012. Comparative transcriptomics of the saprobic and parasitic growth phases in Coccidioides spp. PloS one 7:e41034.

28. Greiner S, Lehwark P, Bock R. 2019. OrganellarGenomeDRAW (OGDRAW) version 1.3.1: expanded toolkit for the graphical visualization of organellar genomes. Nucleic Acids Res 47:W59-W64.

29. Li H, Durbin R. 2009. Fast and accurate short read alignment with Burrows-Wheeler transform. Bioinformatics 25:1754-60. 
30. McKenna A, Hanna M, Banks E, Sivachenko A, Cibulskis K, Kernytsky A, Garimella K, Altshuler D, Gabriel S, Daly M, DePristo MA. 2010. The Genome Analysis Toolkit: a MapReduce framework for analyzing next-generation DNA sequencing data. Genome Res 20:1297-303.

31. Kurtz S, Phillippy A, Delcher AL, Smoot M, Shumway M, Antonescu C, Salzberg SL. 2004. Versatile and open software for comparing large genomes. Genome Biol 5:R12.

32. Nguyen LT, Schmidt HA, von Haeseler A, Minh BQ. 2015. IQ-TREE: a fast and effective stochastic algorithm for estimating maximum-likelihood phylogenies. Mol Biol Evol 32:26874 .

33. Kalyaanamoorthy S, Minh BQ, Wong TKF, von Haeseler A, Jermiin LS. 2017. ModelFinder:

34. Minh BQ, Nguyen MA, von Haeseler A. 2013. Ultrafast approximation for phylogenetic

35. Puigbò P, Garcia-Vallvé S, McInerney JO. 2007. TOPD/FMTS: a new software to compare

36. Van Dyke MCC, Teixeira MM, Barker BM. 2019. Fantastic yeasts and where to find them:

37. Schroder MS, Martinez de San Vicente K, Prandini TH, Hammel S, Higgins DG, Bagagli E,

38. van de Sande WW. 2012. Phylogenetic analysis of the complete mitochondrial genome of

39. Ballard JW, Whitlock MC. 2004. The incomplete natural history of mitochondria. Mol Ecol

40. Sota T, Vogler AP. 2001. Incongruence of mitochondrial and nuclear gene trees in the Carabid beetles Ohomopterus. Syst Biol 50:39-59.

42. Turissini DA, Gomez OM, Teixeira MM, McEwen JG, Matute DR. 2017. Species boundaries

43. Pryszcz LP, Nemeth T, Saus E, Ksiezopolska E, Hegedusova E, Nosek J, Wolfe KH, Gacser A, Gabaldon T. 2015. The Genomic Aftermath of Hybridization in the Opportunistic Pathogen Candida metapsilosis. PLoS Genet 11:e1005626.

44. Ma H, May RC. 2010. Mitochondria and the regulation of hypervirulence in the fatal fungal outbreak on Vancouver Island. Virulence 1:197-201.

45. Depotter JRL, Beveren Fv, C.M. van den Berg G, Wood TA, Thomma BPHJ, Seidl MF. 2018. Nuclear and mitochondrial genomes of the hybrid fungal plant pathogen $<$ em $>$ Verticillium longisporum $</$ em $>$ display a mosaic structure. bioRxiv doi:10.1101/249565:249565. 
424 46. Keuler R, Garretson A, Saunders T, Erickson RJ, St. Andre N, Grewe F, Smith H, Lumbsch HT, Huang J-P, St. Clair LL, Leavitt SD. 2020. Genome-scale data reveal the role of

427 47. Taylor JW, Barker BM. 2019. The endozoan, small-mammal reservoir hypothesis and the life cycle of Coccidioides species. Med Mycol 57:S16-S20.

48. Li XC, Peris D, Hittinger CT, Sia EA, Fay JC. 2019. Mitochondria-encoded genes contribute to evolution of heat and cold tolerance in yeast. Sci Adv 5:eaav1848.

431 49. Baker EP, Peris D, Moriarty RV, Li XC, Fay JC, Hittinger CT. 2019. Mitochondrial DNA and temperature tolerance in lager yeasts. Sci Adv 5:eaav1869. 


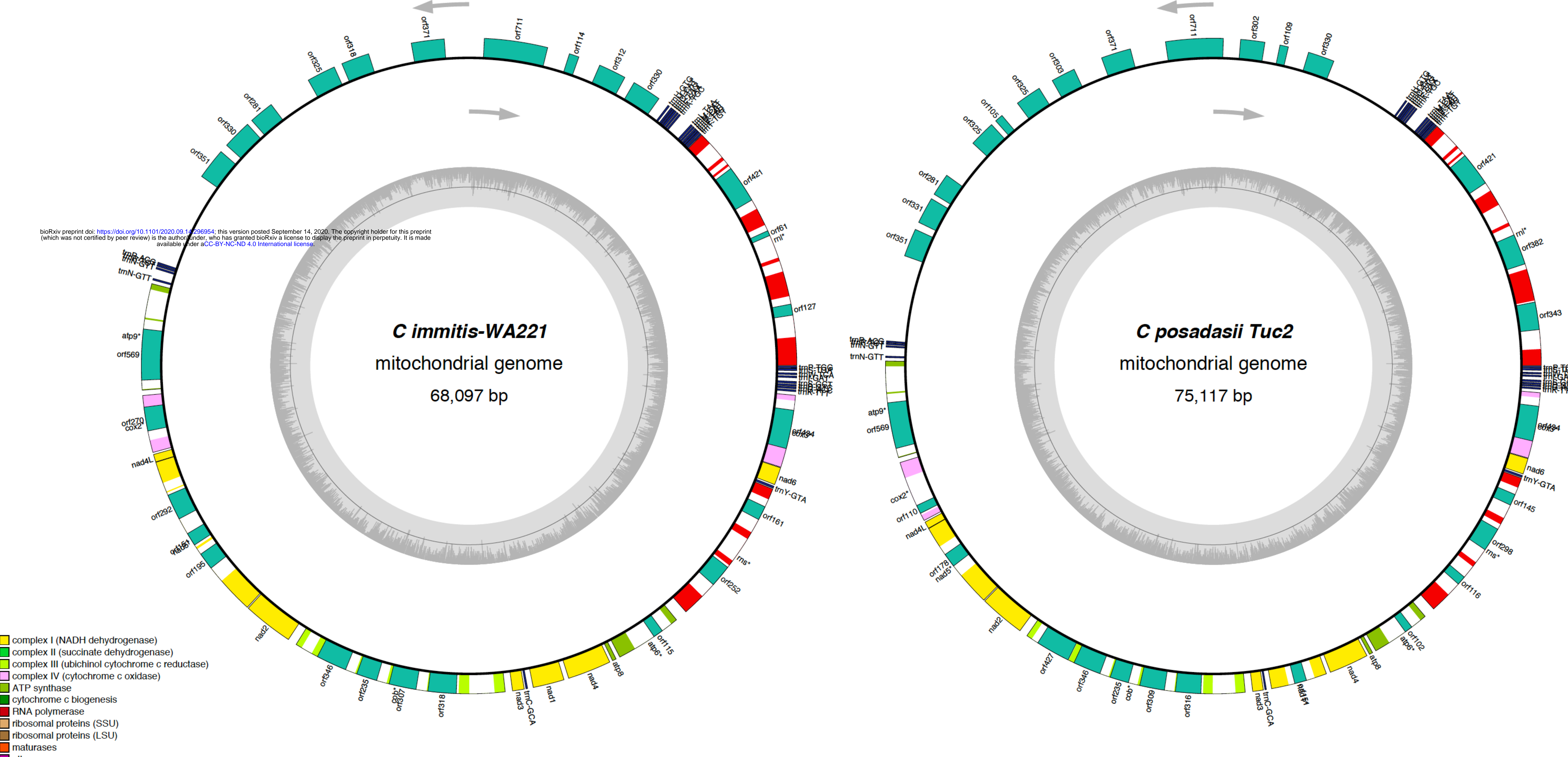


C. immitis

WA_221

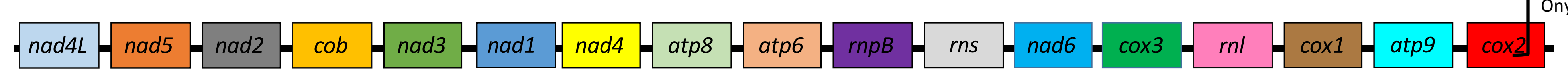

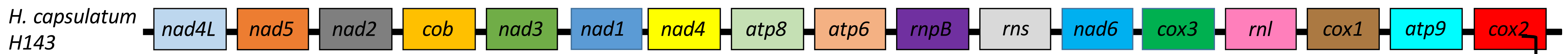

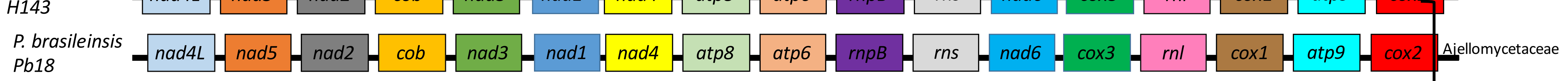

B. dermatitidis

T. rubrum

CBS 118892

E. floccosum

ATCC 26072

A. apis

ARSEF 7405

cob nad3 nad1 nad4

$\operatorname{nad} 4$ atp8

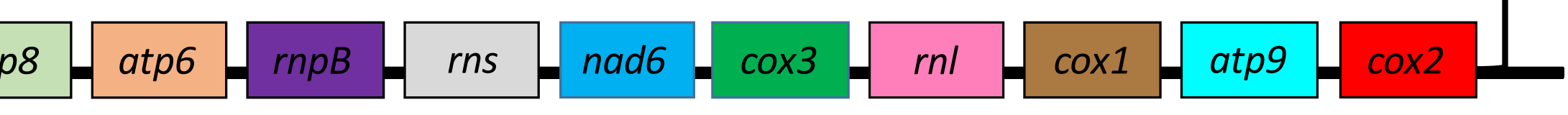

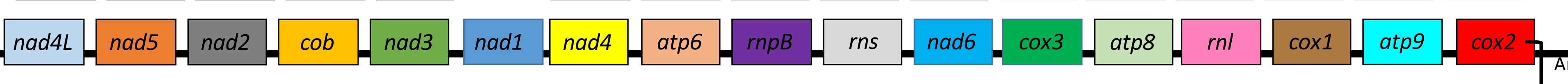

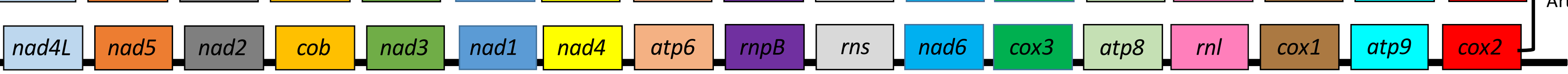

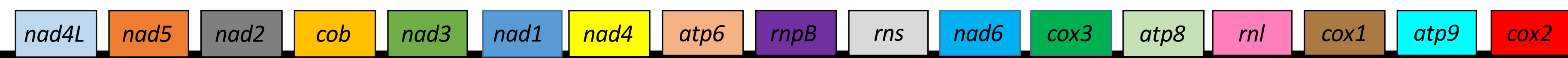




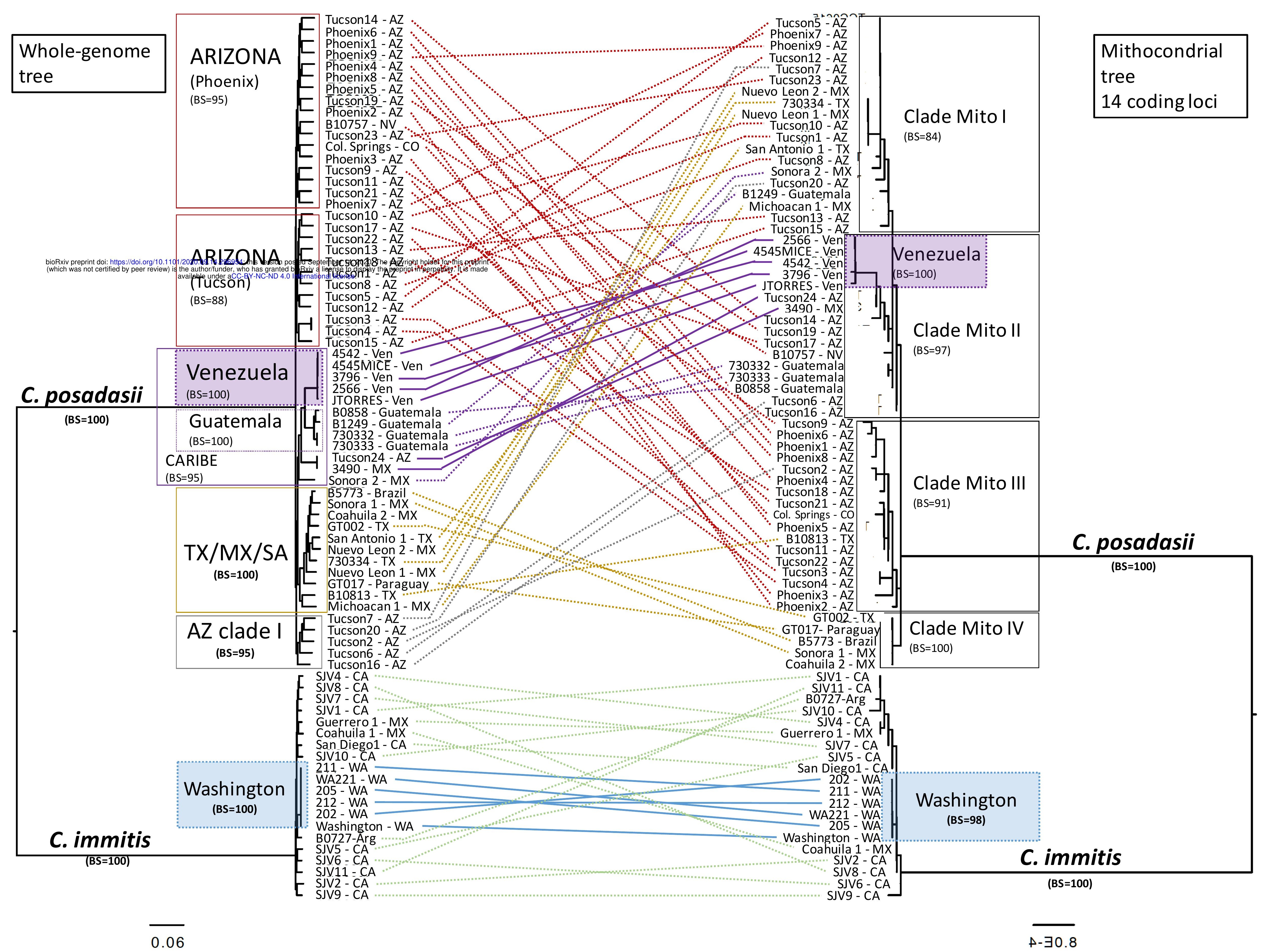

